\title{
Modified Conjugate Gradient Algorithms for Gram Matrix Inversion of Massive MIMO Downlink Linear Precoding
}

\author{
D.Subitha, J.M.Mathana, J.S.Leena Jasmine,R.Vani
}

\begin{abstract}
This paper deals with various low complexity algorithms for higher order matrix inversion involved in massive MIMO system precoder design. The performance of massive MIMO systems is optimized by the process of precoding which is divided into linear and nonlinear. Nonlinear precoding techniques are most complex precoding techniques irrespective of its performance. Hence, linear precoding is generally preferred in which the complexity is mainly contributed by matrix inversion algorithm. To solve this issue, Krylov subspace algorithm such as Conjugate Gradient (CG) was considered to be the best choice of replacement for exact matrix inversions. But CG enforces a condition that the matrix needs to be Symmetric Positive Definite (SPD). If the matrix to be inverted is asymmetric then CG fails to converge. Hence in this paper, a novel approach for the low complexity inversion of asymmetric matrices is proposed by applying two different versions of $C G$ algorithms- Conjugate Gradient Squared (CGS) and Bi-conjugate Gradient (Bi-CG). The convergence behavior and BER performance of these two algorithms are compared with the existing $C G$ algorithm. The results show that these two algorithms outperform CG in terms of convergence speed and relative residue.
\end{abstract}

Keywords: Massive MIMO, Linear precoding, Krylov subspace algorithms, convergence speed, relative residue.

\section{INTRODUCTION}

The ongoing research in wireless era is mainly focusing on developing $5 \mathrm{G}$ wireless standards since they provide the application in all the fields such as agriculture, e-health etc. So as to support standards like this, technologies such as Multiple Input Multiple Output (MIMO), massive MIMO and millimeter wave MIMO are very much helpful. These technologies help to improve data rate, spectrum efficiency, quality and reliability. These benefits set the basis for $5 \mathrm{G}$ networks [1]. The traditional approach to obtain these targets is by adding the number of Base Stations (BS), thereby reducing the area of coverage of each BS. However this suffers with increased interference between mobile terminals and base stations of adjacent cells, since the distance between the co-cells reduces. Moreover having larger number of base

Revised Version Manuscript Received on 10 September, 2019.

D.Subitha, Assistant Professor, Department of ECE, Saveetha School for Engineering, Chennai, Tamil Nadu, India.(Email: subitharaj01@ gmail.com)

J.M.Mathana, Principal, Chennai Institute of Technology Chennai, Tamil Nadu, India.(Email: jm.mathana@gmail.com)

.S.Leena Jasmine, Associate Professor, Department of ECE,Velammal Engineering College Chennai, Tamil Nadu, India.(Email: leena@velammal.edu.in)

R.Vani, Associate Professor, Department of ECE, SRM University, Chennai, Tamil Nadu, India.(Email: vanigowtham18@gmail.com) stations increases the frequency of handover which causes more burdens to the system.

To solve the above stated issues, MIMO is proved to be a capable alternate for increasing the spectral efficiency and capacity of the wireless systems. It uses large antenna array in the order of Hundreds whereas the existing uses only in the order of Tens. By means of this large scale set up massive MIMO provides excessive benefits in terms of capacity, data rate and spectrum efficiency. Thus, this offers a more promising solution for the ongoing research and developments in the field of $5 \mathrm{G}$ by increasing the efficiency to hundred times which could be possible only by hundred numbers of base stations in ordinary MIMO. Theoretically making the number of antennas to infinity reduces Inter Cell Interference (ICI) and small scale fading effects. The fact behind the concept of Large Scale Antenna Array is, as the number of antenna elements increases the orthogonally between data transmitted by the antenna elements increases; thereby reducing ICI [2]-[5].But this results in Multi-User Interference (MUI) which can be mitigated by using suitable precoding techniques at the transmitter side. To reach the complete potential of large scale MIMO system it is essential to do efficient precoding. Transmit side precoding is a generalization of beamforming that facilitates the transmission of multi-stream of data intended for $\mathrm{K}$ number of mobile users through $\mathrm{M}$ transmit antenna elements at the BS. The data symbols transmitted from each antenna element is the weighted (precoder) sum of all user data. Precoding techniques, in general are of two types; linear precoding and nonlinear precoding. In [6], the sum-rate performance of linear precoding techniques such as ZF and MMSE are evaluated under the scenario of residential area with a BS equipped with 128 antennas and for 2 mobile users. This ZF and MMSE algorithms result in a near optimal performance of up to $98 \%$ of the optimal non-linear Dirty Paper Coding (DPC) precoding algorithm. For regular MIMO systems both linear and non linear precoding can be used. But for massive MIMO systems the use of non linear precoding techniques is highly impossible as the complexity of the algorithms amplify with the large antenna set up. Thus it is highly desirable to opt for low complexity linear precoding techniques such as ZF and MMSE for Large Scale Antenna Arrays [13]. The performance of these linear precoding techniques are highly relying on the ability to compute the 


\section{MODIFIED CONJUGATE GRADIENT ALGORITHMS FOR GRAM MATRIX INVERSION OF MASSIVE MIMO DOWNLINK LINEAR PRECODING}

matrix inversions faster at the speed equal to the Doppler rate. Here the matrix to be inverted is the Gram matrix of size $K \times K$ whose dimension increases as the number of user antennas ( $K$ ) increases.

An approximate matrix inversion algorithm namely Neuman series expansion was proposed in [7]-[9] to compute Gram matrix inversion faster. In [10], CG algorithm was used for approximate matrix inversions which results better than Neuman series. These algorithms, compute matrix inversion approximations with low computational complexity than the exact inversion algorithms.

CG method of precoding fails when the matrix to be inverted is asymmetric. Hence in this paper, we propose two modified CG algorithms to handle this issue of finding large matrix inversions faster, especially when the matrix is asymmetric. The rest of the paper is organized as follows: the system model of the ZF precoder in section II, low complexity matrix inversion algorithms are proposed in section III and the simulation results are discussed in section IV.

\section{ZERO-FORCING PRECODING - SYSTEM MODEL}

In this section, we intended to focus only on ZF precoding at the downlink of a massive MIMO, since it strikes a balance between complexity and performance. Consider a scenario of number of antenna elements that serves $K$ number of mobile users residing in the same cell. The data symbol vector intended for $K$ users is given as

$$
\mathbf{s}=\left[\begin{array}{llll}
s_{1} & s_{2} & \ldots & s_{K}
\end{array}\right]
$$

where $s_{i}, i=1,2, \ldots . k$ is the data symbol corresponds to the $i^{\text {th }}$ user.

This vector $\mathbf{S} \in C^{K \times 1}$ has to be transmitted over $\mathrm{M}$ number of antennas, thus producing the transmitted data vector $\mathbf{x} \in C^{M \times 1}$ as given below

$$
\mathbf{x}=\mathbf{W} \mathbf{s}
$$

where $\mathbf{W}$ is the mapping vector that transforms $\mathbf{S}$ into $\mathbf{x}$, called as precoding weight matrix of size $M \times K$. Here the precoder matrix $\mathbf{W}$ is given as

$$
\mathbf{W}=\left[\begin{array}{ccc}
w_{11} & \ldots & w_{1 K} \\
\vdots & \ddots & \vdots \\
w_{M 1} & \cdots & w_{M K}
\end{array}\right]
$$

The element of the matrix, $w_{i j}$ refers to the weight of the link connecting $\mathrm{i}^{\text {th }}$ transmitting antenna of the BS and antenna of the $\mathrm{j}^{\text {th }}$ user. These elements are calculated in such a way that the transmitted data is received only by the intended user, causing minimal interference to other users. The precoding matrix $\mathbf{W}$ is calculated for every user terminal by forcing every other's to zero. As referred in [5] the precoding matrix $\mathbf{W}$ is expressed as single cell, in which the BS is equipped with an array of $M$

$$
\mathbf{W}=\mathbf{H}^{H}\left(\mathbf{H H}^{H}\right)^{-1}
$$

where $\mathbf{H} \in C^{K \times M}$ is the channel matrix of dimension $K \times M$.This Channel knowledge is obtained by the transmission of uplink pilot transmissions that acquires instantaneous Channel State Information (CSI) at each BS. The above equation can be represented using Gram matrix $\mathbf{G}$ as

$$
\mathbf{W}=\mathbf{H}^{H} \mathbf{G}^{-1}
$$

Substituting the above equation of $\mathbf{W}$ in (2) yields

$$
\mathbf{x}=\mathbf{H}^{H} \mathbf{G}^{-1} \mathbf{s}
$$

The transmitted data vector $\mathbf{X}$ is then sent over the channel where complex white noise $\mathbf{n} \in C^{K \times 1}$ is added, that yields

$$
\begin{gathered}
\mathbf{y}=\mathbf{H x}+\mathbf{n} \\
\text { where } \quad \mathbf{y}=\left[\begin{array}{lllll}
y_{1} & y_{2} & \cdots & y_{K-1} & y_{K}
\end{array}\right] \quad C^{K \times 1}
\end{gathered}
$$
corresponds to the symbol vector received by the $K$ user antennas for detection.

The Fig.1 shows the steps involved in the generation of precoding matrix $\mathbf{W}$ from the instantaneous channel matrix H.

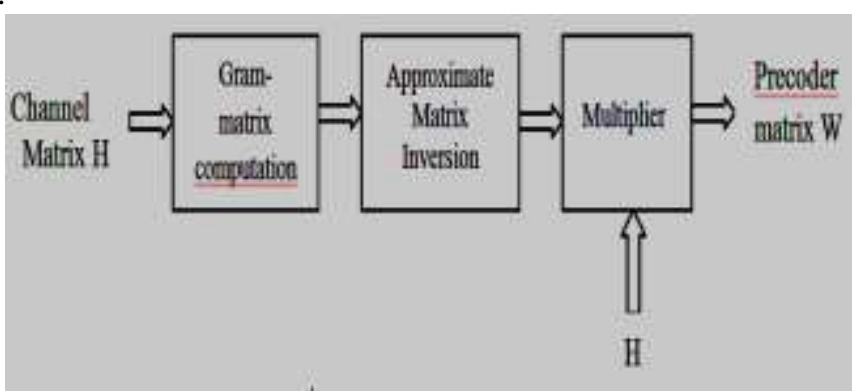

Fig. 1. Block diagram of ZF precoder

The above Figure illustrates the three basic steps in the computation of the Gram matrix $\mathbf{G}$. They are a) Computation of Gram matrix $\mathbf{G}$ b) Gram matrix Inversion $\mathbf{G}^{-1}$ c) Multiplier to find $\mathbf{G}^{-1} \mathbf{H}^{H}$. The implementation of the above structure in hardware requires less complex operations for stp1 and step 3. But, implementing the inverse module is the more complex task since multiplier modules can be easily implemented using hardware MAC units. But the inverse computation module requires division operation that is highly infeasible in hardware. Hence finding inverse of a matrix is computationally challenging; particularly, when the matrix to be inverted is quite high. In order to make $\mathrm{ZF}$ precoding less complex, Krylov subspace iterative algorithms such as CG[10] and its variant algorithms proposed in this paper, are used in place of exact inversions for computing $\mathbf{G}^{-1}$.In general, iterative algorithms are preferred over other matrix inversion algorithms due to their adaptability to hardware in supporting parallel processing.

\section{KRYLOV SUBSPACE ITERATIVE ALGORITHMS FOR ASYMMETRIC GRAM MATRIX INVERSION}

In our case, we consider the channel simulated using WINNER phase II channel model [11] is complex and random. For a system of $M$ transmit antennas at the BS and 
$K$ number of user terminals, $\mathbf{H}$ is a $K \times M$ complex matrix. Hence the Gram matrix $\mathbf{G}$ is asymmetric with all its diagonal elements real and the entries in the upper triangular matrix are the complex conjugate of the corresponding entry in the lower triangular which will be of the form given in below

$$
\mathbf{G}=\mathbf{H H}^{H}=\left[\begin{array}{cccc}
g_{11} & g_{12} & \cdots & g_{1 K} \\
g_{12}^{*} & g_{22} & \cdots & g_{2 K} \\
\vdots & \vdots & \ddots & \vdots \\
g_{1 K}^{*} & g_{2 K}^{*} & \cdots & g_{K K}
\end{array}\right]
$$

$\mathrm{CG}$ method of precoding is not useful to find the inverse of the matrix in the above form, since it is asymmetric. Hence in this paper, we propose a novel approach for the low complexity inversion of asymmetric matrices by applying two different versions of CG algorithms- Conjugate Gradient Squared (CGS) and Bi-conjugate Gradient (Bi-CG) where all of them are Krylov subspace algorithms.

\section{A. Krylov Subspace Iterative Algorithms}

Out of many algorithms available for solving linear systems, Krylov subspace iterative algorithms are the most popular iterative technique suitable for solving linear systems of larger sizes. A linear system of the form $\mathbf{G x}=\mathbf{b}$ is solved by approximating $\mathbf{X}$ with the optimization function given below,

$$
\widehat{\mathbf{x}}=\arg \min \|\mathbf{b}-\mathbf{G} \mathbf{x}\|
$$

where $\mathbf{G}$ is a Positive Definite Gram matrix to be inverted, $\mathbf{b}$ and $\mathbf{x}$ are column vectors. The Krylov subspace algorithm computes a good approximate at each iteration step k, by searching in the subspace $\kappa_{m}(\mathbf{G}, \mathbf{b})$ spanned by $\mathbf{G}$ and $\mathbf{b}$.The following subspace is called Krylov subspace

$$
\kappa_{m}(\mathbf{G}, \mathbf{b})=\operatorname{span}\left\{\mathbf{b}, \mathbf{G b}, \mathbf{G}^{2} \mathbf{b} \cdots \cdots, \mathbf{G}^{k-1} \mathbf{b}\right\}
$$

CG method is the first Krylov subspace method which is designed only for solving SPD linear systems, where the coefficient matrix $\mathbf{G}$ satisfies the conditions (i) $\mathbf{H}^{H}=\mathbf{G}$ (ii)

$\mathbf{a}^{H} \mathbf{G a}>0$ for $\mathbf{a} \neq 0$ []. CG is considered to be the best that iteratively computes the solution $\widehat{\mathbf{x}}=\mathbf{G}^{-1} \mathbf{b}$ with each iteration requiring low computational complexity. Since the channel matrices $\mathbf{H}$ are well conditioned and diagonally dominant, the popular Krylov subspace algorithm such as CG works well and provides speedy convergence with at most $K$ number of iterations at which the relative residue is zero and can even be terminated earlier than $\mathrm{K}$ with a tolerable residue. But CG enforces a condition that the matrix $\mathbf{G}$ needs to be SPD. If the matrix to be inverted is asymmetric then CG fails to converge.

\section{B. Bi-Conjugate Gradient Algorithm (Bi-CG)}

When the matrix to be inverted $\mathbf{G}$ is asymmetrical and if $\mathbf{G}^{H}$ can be found, then $\mathrm{Bi}-\mathrm{CG}$ is the best Krylov subspace algorithm. Starting with the random initial guess $\mathbf{x}_{0}$, the algorithm refines the solution of $\mathbf{X}$ in every step. The iteration starts with an initial residue $\mathbf{r}_{0}=\mathbf{b}$ and the initial solution $\mathbf{x}_{0}=0$. At every iteration, the solution is updated in the search direction $\mathbf{d}_{k}$ by the step length $\alpha_{k}$. The residue is also recursively reduced in the same direction by the amount of $\alpha_{k}$ and $\mathbf{G}$.

For the downlink BS, to obtain the transmitted symbol vector $\mathbf{X}$ given in (6) using algorithm 1, the following substitutions has to be made; $\mathbf{G}=\mathbf{H} \mathbf{H}^{H}, \mathbf{b}=\mathbf{s}$. At the end of $K{ }^{\text {th }}$ iteration, the solution obtained is $\widehat{\mathbf{x}}=\mathbf{x}_{K}=\mathbf{G}^{-1} \mathbf{S}$. To obtain the transmitted symbol vector $\mathbf{X}$ in (6), the solution obtained at the final iteration need to be multiplied with $\mathbf{H}^{H}$.

\section{Algorithm 1: Bi-CG for asymmetric Gram matrix} inversion

// System to be solved: $\widehat{\mathbf{x}}=\mathbf{G}^{-1} \mathbf{b}$

// Input: $\mathbf{G}=\mathbf{H H}^{H}, \mathbf{b}=\mathbf{s}$

// Initial Guess: For $k=0$

$$
\mathbf{x}_{0}=0 ; \mathbf{r}_{0}=\mathbf{b} ; \mathbf{d}_{0}=\mathbf{r}_{0}
$$

// Iteration starts: for $\mathrm{k}=1,2 \ldots . \mathrm{K}$

// Step length

$\alpha_{k}=\frac{\mathbf{r}_{k-1}^{H} \mathbf{r}_{k-1}}{\mathbf{d}_{k-1}^{H} \mathbf{G d}_{k-1}}$

//Approximate solution:

$\mathbf{x}_{k}=\mathbf{x}_{k-1}+\alpha_{k} \mathbf{d}_{k-1}$

// Residual correction

$\mathbf{r}_{k}=\mathbf{r}_{k-1}-\alpha_{k} \mathbf{G d}_{k-1}$

// Improvement parameters

$$
\beta_{k}=\frac{\mathbf{r}_{k}^{H} \mathbf{r}_{k}}{\mathbf{r}_{k-1}^{H} \mathbf{r}_{k-1}}
$$

// Search directional vector

$\mathbf{d}_{k}=\mathbf{r}_{k}+\beta_{k} \mathbf{d}_{k-1}$

// Relative residue calculation

$$
R_{k}=\frac{\left\|\mathbf{b}^{T}-\mathbf{G} \mathbf{x}_{k}\right\|}{\|\mathbf{b}\|}
$$

$$
\begin{aligned}
& \text { // Transmitted symbol vector calculation } \\
& \mathbf{x}=\mathbf{H}^{H} \mathbf{x}_{K}
\end{aligned}
$$

Finally the relative residue $R_{k}$, which is the parameter used for comparing the residue obtained at the end of iteration with the initial residue is

$$
R_{k}=\frac{\operatorname{norm}\left(\mathbf{b}^{T}-\mathbf{G} \mathbf{x}_{k}\right)}{\operatorname{norm}(\mathbf{b})}
$$

Using this parameter, the convergence speed and accuracy of the iterative algorithm is analyzed. 


\section{MODIFIED CONJUGATE GRADIENT ALGORITHMS FOR GRAM MATRIX INVERSION OF MASSIVE MIMO DOWNLINK LINEAR PRECODING}

\section{Conjugate Gradient Squared Algorithm (CGS)}

The major drawback of BCG is computing the transpose of $\mathbf{G}$. This can be easily eliminated by CGS algorithm and to gain faster convergence for roughly at the same computational cost. Starting with a initial residue $\mathbf{r}_{0}=\mathbf{b}$, Bi-CG algorithm evaluates the residue at the $k^{\text {th }}$ iteration as given below

$$
\mathbf{r}_{k}=P_{i}(\mathbf{G}) \mathbf{r}_{0}
$$

This suggests that, if $P_{i}(\mathbf{G})$ reduces $\mathbf{r}_{0}$ to a smaller value $\mathbf{r}_{k}$; where $P_{i}(\mathbf{G})$ is the polynomial in $\mathbf{G}$ called as contraction operator. Then it is considered to apply this contraction operator $P_{i}(\mathbf{G})$ twice to compute $P_{i}^{2}(\mathbf{G}) \mathbf{r}_{0}$ .Hence this algorithm is named as Conjugate Gradient Squared algorithm (CGS)[]. Since the contraction operation is applied twice the convergence speed is about twice as fast as for the Bi-CG method. At the same time, the number of computations required per iteration by CGS is almost same as the Bi-CG method except for the computation of $\mathbf{G}^{H}$. That is, in calculating $\alpha_{k}$ in CGS involves only $\mathbf{r}_{0}^{H}$ where $\mathbf{r}_{0}=\mathbf{b}$. But in Bi-CG, it is calculated using $\mathbf{r}_{k-1}^{H}$ which involves the residue of previous iteration for which $\mathbf{G}^{H}$ must be known. Hence in circumstances, where the computation of $\mathbf{G}^{H}$ is impractical CGS may be attractive. But the drawback of CGS is irregular convergence behavior.

Both the algorithms are compared based on the relative residue $R_{k}$ calculated at the end of iteration using $\mathbf{G}, \mathbf{b}$ and the solution $\mathbf{x}_{k}$.

\section{Algorithm 1: CGS for asymmetric Gram matrix inversion}

$$
\begin{aligned}
& \text { // System to be solved: } \widehat{\mathbf{x}}=\mathbf{G}^{-1} \mathbf{b} \\
& \text { // Input: } \mathbf{G}=\mathbf{H} \mathbf{H}^{H}, \mathbf{b}=\mathbf{S} \\
& \text { // Initial Guess: For } k=0 \\
& \quad \mathbf{x}_{0}=0 ; \mathbf{r}_{0}=\mathbf{b} ; \mathbf{d}_{0}=\mathbf{r}_{0} ; \mathbf{u}_{0}=\mathbf{b}
\end{aligned}
$$

// Iteration starts: for $\mathrm{k}=1,2 \ldots . \mathrm{K}$

// Step length

$$
\begin{aligned}
& \alpha_{k}=\frac{\mathbf{r}_{0}^{H} \mathbf{r}_{k-1}}{\mathbf{r}_{0}^{H} \mathbf{G d}_{k-1}} \\
& \mathbf{q}_{k-1}=\mathbf{u}_{k-1}-\alpha_{k} \mathbf{G d}_{k-1}
\end{aligned}
$$

//Approximate solution:

$$
\mathbf{x}_{k}=\mathbf{x}_{k-1}+\alpha_{k}\left(\mathbf{u}_{k-1}+\mathbf{q}_{k-1}\right)
$$

// Residual correction

$$
\mathbf{r}_{k}=\mathbf{r}_{k-1}-\alpha_{k} \mathbf{G}\left(\mathbf{u}_{k-1}+\mathbf{q}_{k-1}\right)
$$

// Improvement

$$
\begin{aligned}
\beta_{k} & =\frac{\mathbf{r}_{0}^{H} \mathbf{r}_{k}}{\mathbf{r}_{0}^{H} \mathbf{r}_{k-1}} \\
\mathbf{u}_{k} & =\mathbf{r}_{k}+\beta_{k} \mathbf{q}_{k-1}
\end{aligned}
$$

$\mathbf{d}_{k}=\mathbf{u}_{k}+\beta_{k}\left(\mathbf{q}_{k-1}+\beta_{k} \mathbf{d}_{k-1}\right)$

// Relative residue calculation

$$
\begin{aligned}
& R_{k}=\frac{\left\|\mathbf{b}^{T}-\mathbf{G} \mathbf{x}_{k}\right\|}{\|\mathbf{b}\|} \\
& / / \quad \text { Transmitted symbol vector calculation } \\
& \mathbf{x}=\mathbf{H}^{H} \mathbf{x}_{K}
\end{aligned}
$$

\section{SIMULATION RESULTS}

In this section, we present the simulation results of the proposed algorithms (CGS, Bi-CG) for computing large size matrix inversions in downlink precoding and compared them with the CG algorithm [10].

\section{A. Convergence Behavior}

For the downlink precoding with $M$ antennas at the BS serving $K$ number of users simultaneously requires the estimation of channel matrix $\mathbf{H}$ of size $K \times M$.For most of the prior work in massive-MIMO, Time-Division Duplexe (TDD) mode is assumed where the downlink channel is the transpose of the uplink channel matrix. Hence we assume that the BS has the knowledge of the downlink channel matrix of all users based on uplink pilot transmission. With this we simulate purely stochastic complex channel H using WINNER phase-II model, whose elements are zero mean, unit variance complex Gaussian variables. The simulations are carried out in a rich scattering scenario where MS is surrounded by large number of scatterers.

The simulation results given in Fig. 2 and Fig. 3 show the convergence behavior of the algorithms in computing $\mathbf{G}^{-1}$ for the downlink system with different number of user antennas $K$ being served simultaneously by the single BS. For the inversion of asymmetric Gram matrix $\mathbf{G}$, the Fig. 2 (a) and (b) clearly show that CG fails to converge with the largest relative residue. But Bi-CG and CGS holds good results with comparatively lesser relative residue in each step. For the same number of user antennas $K=3$ and $K=15$, Fig. 3(a) and (b) shows the comparison of all three algorithms for the inversion of symmetric $\mathbf{G}$. From these results, it is evident that $\mathrm{CG}$ is better than the other two methods for the inversion of symmetric $\mathbf{G}$. This clearly shows the inability of $\mathrm{CG}$ under asymmetric cases.

Fig. 2, also illustrates the convergence speed of CGS and $\mathrm{Bi}-\mathrm{CG}$ and it is proven that CGS converges almost twice the speed of Bi-CG since it start with low initial residue. At every iteration $\mathrm{k}=1,2 \ldots . . K$, since the residue of CGS is almost two times lesser than Bi-CG, it is possible to stop much before $K^{\text {th }}$ iteration with a tolerable relative residue. Thus the computations required are lesser for CGS. But the drawback of CGS is, its irregular convergence behavior for larger $K$ as in Fig .2(b) and hence requires good preconditioners whereas Bi-CG holds consistent results for all values of $K$. 


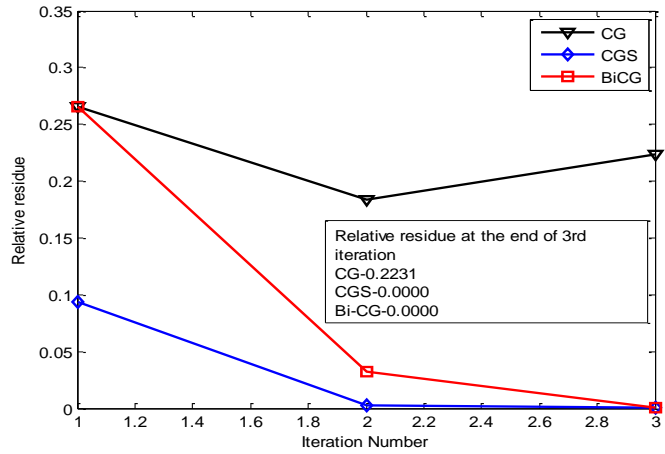

(a) $K=\mathbf{3}$ Users

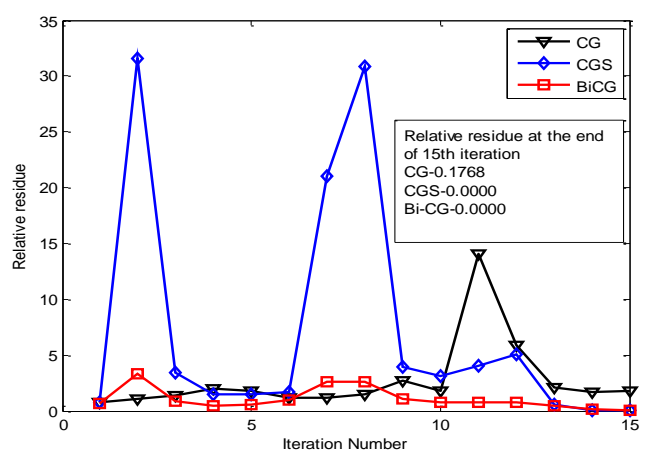

(b) $K=15$ Users

Fig. 2. Comparison of $\mathrm{CG}, \mathrm{CGS}$ and $\mathrm{Bi}-\mathrm{CG}$ algorithms for asymmetric Gram matrix inversion of size $K \times K$ in large Scale MIMO downlink precoding

Fig. 4(a) shows the comparison of the three algorithms over different number of users $K$, for the inversion of asymmetric Gram matrix $\mathbf{G}$ of dimension $K \times K$. Comparisons are made in terms of the residues obtained at the end of $K^{\text {th }}$ step. Results show that, Bi-CG and CGS exhibits almost zero residue whereas residue of $\mathrm{CG}$ progressively increases as $\mathrm{K}$ increases. This simulation is carried out for some sample of channel matrices $\mathbf{H}$ generated randomly by Winner phase-II model with $100 \mathrm{BS}$ antennas serving $K$ mobile users.

Fig 4 (b) reveals that Bi-CG provides consistently minimal residue at the end of $K^{\text {th }}$ step though the relative residues are larger at every $\mathrm{i}^{\text {th }}$ iteration. This is the advantage of Bi-CG over CGS.

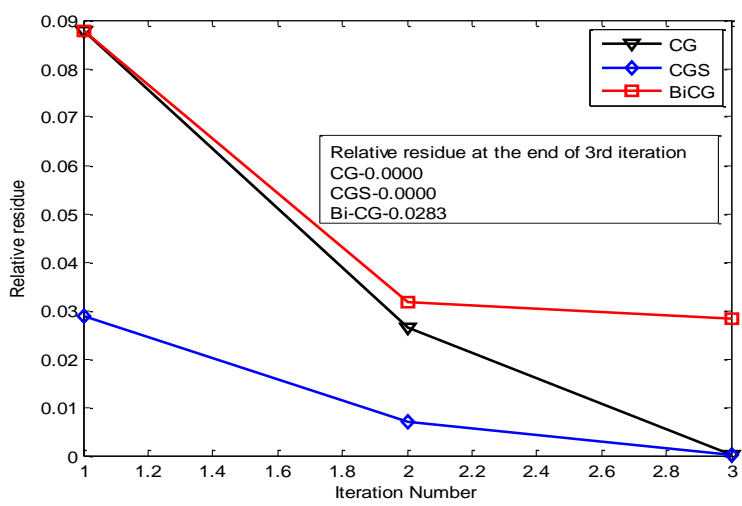

(a) $\mathrm{K}=3$ Users

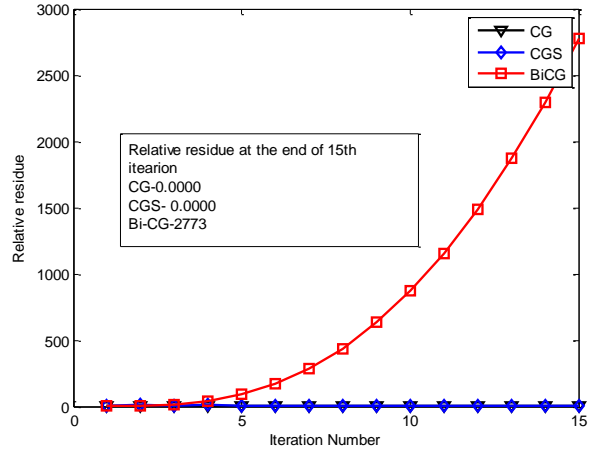

(b) $K=15$ Users

Fig. 3. Comparison of CG, CGS and Bi-CG algorithms for symmetric Gram matrix inversion of size $K \times K$ in large scale MIMO downlink precoding

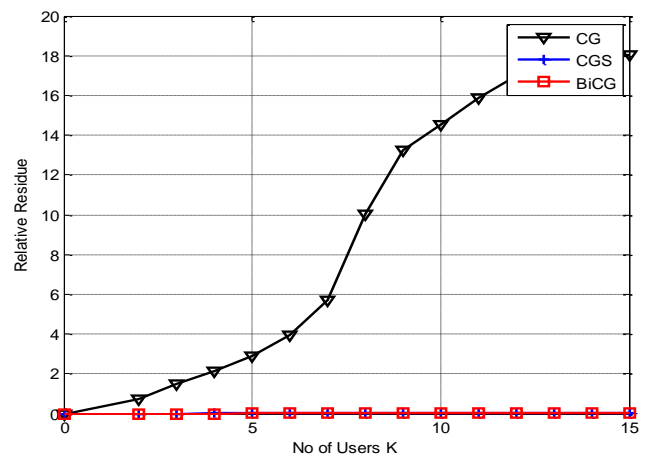

(a) Comparison of CG, CGS and Bi-CG

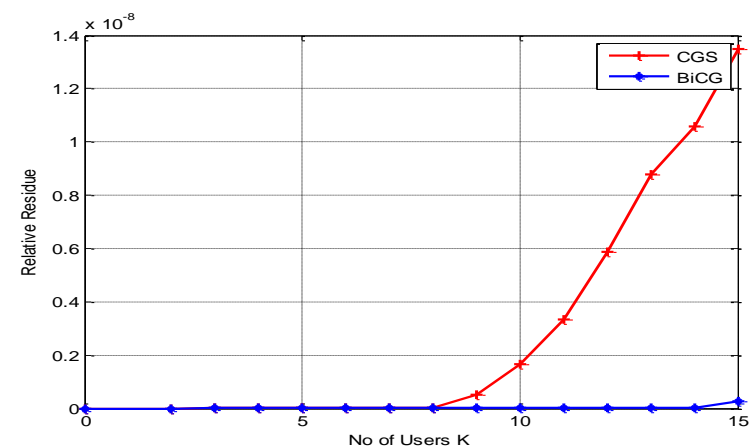

(b) Comparison of CGS and Bi-CG

Fig. 4. Comparison of the $\mathrm{CG}$ algorithms for the computation of $\mathbf{G}^{-1}$ of size $K \times K$ in terms of relative residues calculated at the end of $K^{\text {th }}$ iteration

\section{B. Massive MIMO downlink precoding-BER Performance}

This subsection illustrates the BER performance of the proposed CGS, Bi-CG based precoding in comparison with $\mathrm{CG}$ and exact inversion precoding. The performance of the massive MIMO downlink system is evaluated for BS equipped with $M=100$ and 200 uniform linear antenna array with antenna spacing of $d=\lambda / 2$. 

DOWNLINK LINEAR PRECODING

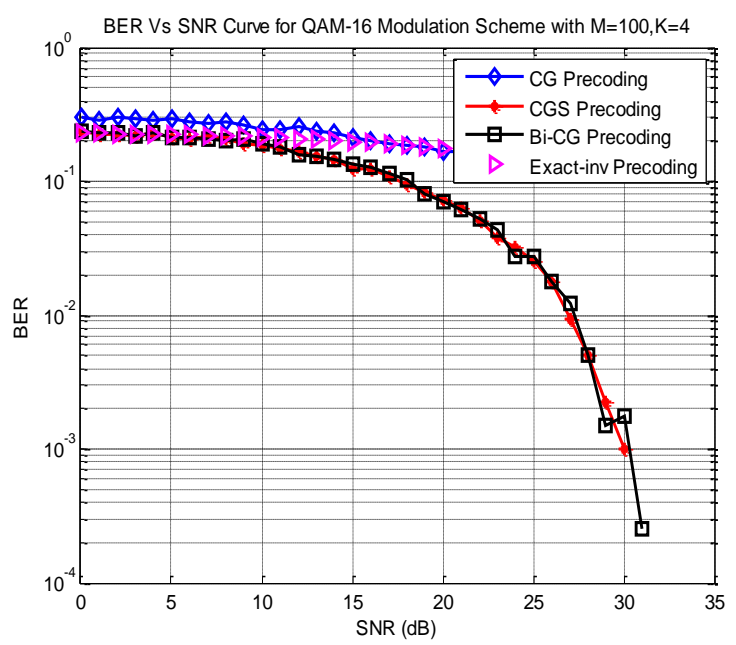

BER Vs SNR Curve for QAM-16 Modulation Scheme with M=200,K=4
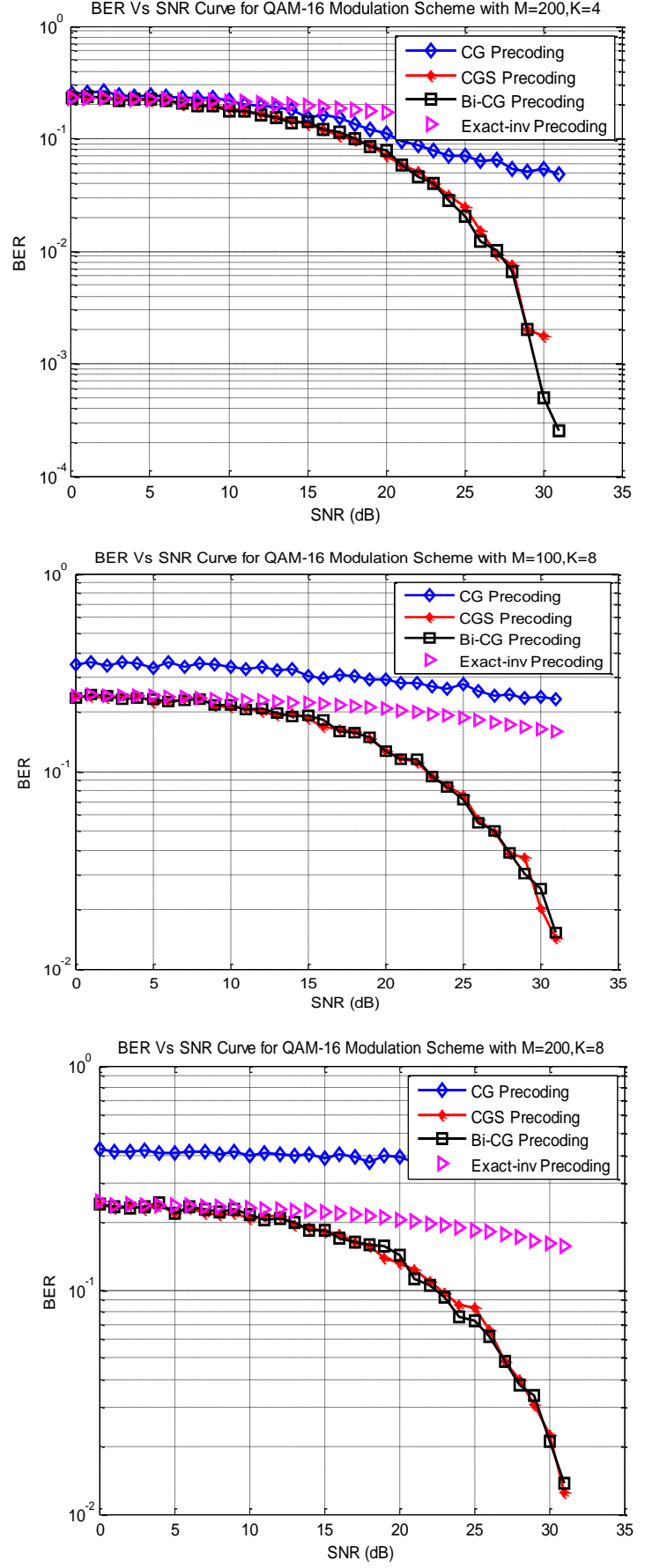

Fig. 5.BER Performance of different precoding algorithms in various $M \times K$ Massive MIMO systems
Transmission sequences are generated randomly and modulated using 16-QAM. The modulated symbols are transmitted through the channel where it is added with zero mean, unit variance Gaussian noise. The transmitted symbol vector is sent through the channel which is simulated using WINNER phase-II model .In Fig. 5 (a) and (b), the BER performance of different precoding algorithms for $K=4$ users is given and Fig. 5(c) and (d) is for $K=8$ users. From the above results, we observe that the proposed CGS and Bi-CG precoding outperforms both $\mathrm{CG}$ and exact inversion precoding under all scenario. The error rate is considerably lesser for CGS and Bi-CG precoding than CG precoding as SNR increases.

\section{CONCLUSION}

We have proposed two algorithms- CGS and Bi-CG for the computation of low complexity inversion of an asymmetric Gram matrix of the massive MIMO downlink linear precoder. The performance is analyzed in terms of convergence rate and relative residue and compared with the existing CG method. For most of the cases CGS provides a relative residue of almost zero in the $(K-1)^{\text {th }}$ iteration itself and so the number of computations required, is much reduced than Bi-CG. Though the convergence speed of CGS is much faster than Bi-CG, the relative residue obtained at the end of $\mathrm{K}$ iterations is much lesser for Bi-CG than CGS. This indicates that $\mathrm{Bi}-\mathrm{CG}$ is more accurate than CGS. Hence we need to strike a balance between rate of convergence and accuracy in choosing any of these two algorithms.

\section{REFERENCES}

1. Huawei Technologies Co., Ltd, "New Air Interface and Radio

2. Access Virtualization" Huawei White Paper, April 2015.

3. T. L. Marzetta, "Noncooperative cellular wireless with unlimited numbers of base station antennas," IEEE Trans. Wireless Commun., vol.9, no.11, pp. 3590-3600, Nov. 2010.

4. H. Huh, G. Caire, H. C. Papadopoulos, and S.A. Ramprashad, "Achieving massive MIMO spectral efficiency with a not-so-large number of antennas," IEEE Trans. Wireless Commun., vol. 11, no. 9, pp. 3266-3239, Sept. 2012

5. F. Rusek, D. Persson, B. K. Lau, E. G. Larsson, T. L. Marzetta, O. Edfors, and F. Tufvesson, "Scaling up MIMO: Opportunities and challenges with very large arrays," IEEE Signal Process. Ma.,, vol. 30, no. 1, pp.40-60, 2013.

6. $\mathrm{Lu} \mathrm{Lu}$, Geoffrey $\mathrm{Ye} \mathrm{Li}, \mathrm{A}$. Lee Swindlehurst, Alexei Ashikhmin, Rui Zhang, "An overview of Massive MIMO: Benefits and challenges," IEEE J. Sel. Topics. vol. 8, no. 5, pp.742-758, 2014

7. X. Gao, O. Edfors, F. Rusek, and F. Tufvesson, "Linear pre-coding performance in measured very-large MIMO channels," in IEEE Veh.Technology Conference (VTC Fall)., Sept. 2011.

8. Prabhu Hemanth, Ove Edfors, Joachim Rodrigues, Liang Liu, and Fredrik Rusek, "Hardware Efficient Approximative Matrix Inversion for Linear Pre-coding in Massive MIMO” IEEE Trans. 2014. 
9. H. Prabhu, J. Rodrigues, O. Edfors, and F.Rusek, "Approximative matrix inverse computations for very-large MIMO and applications to linear pre-coding systems," in IEEE WCNC., Apr. 2013.

10. Michae Wu, Bei Yin, Aida Vosoughi, Christoph Studer, Joseph R. Cavallaro, and Chris Dick "Approximate matrix inversion for high-throughput data detection in the large-scale MIMO uplink," in IEEE Int. Symp.Circuits Syst., ISCAS, May 2013.

11. Bei Yin, Michael Wu, Joseph R. Cavallaro, and Christoph Studer "Conjugate Gradient-based Soft-Output Detection and Precoding in Massive MIMO Systems' ECCS-1408370.

12. L. Hentila, P. Kyosti, M. Kaske, M. Narandzic, and M. Alatossava,"Matlab implementation of the WINNER phase II channel model ver 1.1,'Dec. 2007.

13. Yu, X., Shen, J. C., Zhang, J., \& Letaief, K. B. (2016). Alternating minimization algorithms for hybrid precoding in millimeter wave MIMO systems. IEEE Journal of Selected Topics in Signal Processing, 10(3), 485-500, 2016.

14. Minango, J., Flores, A., Minango, P., \& Ibarra, D. A. Low-Complexity Precoder for Massive MIMO Systems Based on Gram-Schmidt Conjugate Direction Method. IEEE Third Ecuador Technical Chapters Meeting, pp. 1-5, 2018. 